\title{
AGGRESSIVE CLEAR CELL CHONDROSARCOMA OF THE HARD PALATE
}

\author{
AJAY M BHANDARKAR*, BALAKRISHNAN RAMASWAMY
}

Department of Otorhinolaryngology-Head and Neck Surgery, Kasturba Medical College, Manipal University, Manipal, Karnataka, India. Email: bm.ajay@gmail.com

Received: 21 January 2017, Received and Accepted: 09 February 2017

ABSTRACT

Chondrosarcomas constitute 1-3\% of all head and neck tumors. $2 \%$ of all chondrosarcomas are of the "clear cell" variety which follows a locally aggressive, relatively indolent course without predominant invasion of soft tissue. We present a case of a 45 -year-old woman who presented with a aggressive variant of clear cell chondrosarcoma.

Keywords: Chondrosarcoma, Maxillectomy, Clear cell, Hard palate.

(C) 2017 The Authors. Published by Innovare Academic Sciences Pvt Ltd. This is an open access article under the CC BY license (http://creativecommons. org/licenses/by/4. 0/) DOI: http://dx.doi.org/10.22159/ajpcr.2017.v10i5.17216

\section{INTRODUCTION}

Chondrosarcomas constitute $1-3 \%$ of all head and neck tumors. $2 \%$ of all chondrosarcomas are of the "clear cell" variety which follows a locally aggressive, relatively indolent course without predominant invasion of soft tissue [1]. Clear cell chondrosarcoma affects the third to fifth decade predominantly in men. The predominant presenting feature is pain and bleeding in the nasal cavity. Metastasis is rare and commonly occurs to bone, brain, and lung [1]. Radiologically, it presents as an osteolytic lesion with intratumoral calcification with poorly defined margins. Usually, most clear cell chondrosarcomas are intracompartmental without cortical permeation of bone $[1,2]$. Histologically, they show polymorphic large cells with abundant clear cytoplasm and hyperchromatic nuclei without production of chondroid hyaline matrix. Immunohistochemically, it stains positive for S100 and collagen Type II [1,2]. It has to be differentiated from osteosarcoma or osteoblastoma histologically and minor salivary gland carcinoma or squamous cell carcinoma if it occurs in the maxilla. En bloc resection is the treatment of choice as chemoradiation is not effective. Recurrence rate (19\%) is low, and the prognosis is excellent with a good survival rate [1]. We present a case of "aggressive" clear cell chondrosarcoma which did not fit into any criteria of this type of tumor available in literature.

A 45-year-old woman presented with a bleeding mass in the oral cavity with a change of voice of 1 -year duration with pain of 1 -month duration. Diagnostic nasal endoscopy showed a bulge in the nasal cavity floor, and a friable mass was observed predominantly in the left half of hard palate crossing the midline (Fig. 1).

Biopsy done from the mass showed a tumor composed of lobules of malignant cartilage characterized by oval cells with abundant eosinophilic to clear cytoplasm, well-defined cell margin, vesicular nuclei, prominent nucleoli, binucleate to multinucleate, bizarre cells with chondroid matrix, and osteoid (Fig. 2)

The tumor was positive focally for S100 and bcl-2. Histopathologically, it was diagnosed as grade 2 clear cell chondrosarcoma. Contrast enhanced computed tomography scan showed an ill-defined, heterogeneously enhancing mass with few necrotic and calcific areas involving the left hard palate with bony destruction of left maxilla, zygoma and extension into the pterygopalatine fossa and maxillary sinus laterally with medial extension into right nasal cavity and superiorly abutting the left inferior turbinate (Fig. 3).
The tumor was removed en bloc by an extended total maxillectomy with negative margins. The patient presented 4 months after the primary en bloc resection with profuse bleeding from a mass in the oral cavity

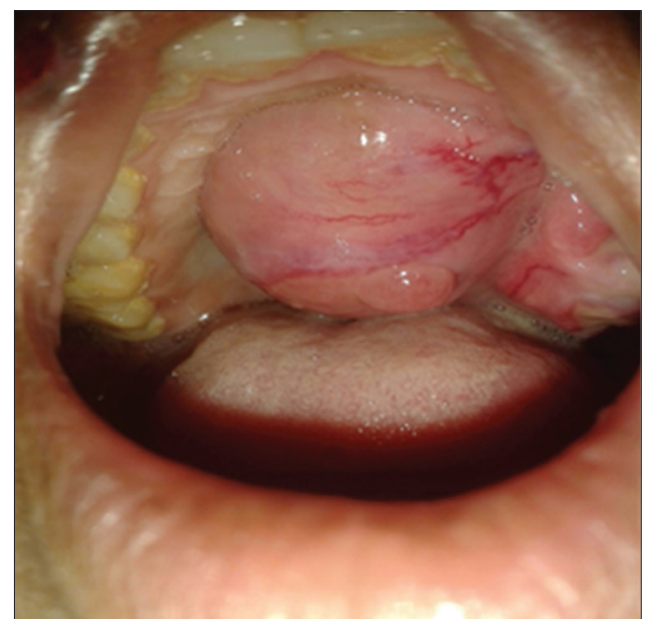

Fig. 1: Intraoral tumor involving the left half of hard palate crossing the midline

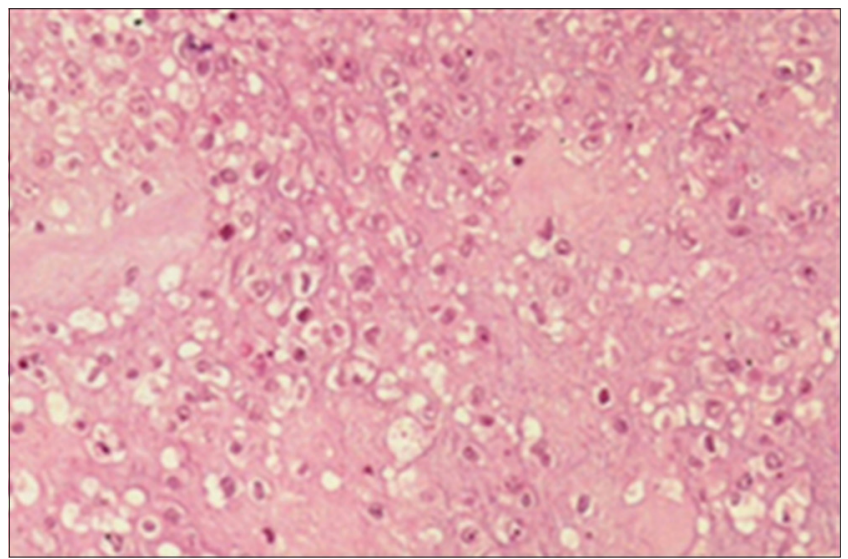

Fig. 2: Hematoxylin and eosin staining showing lobules of malignant cartilage cells 


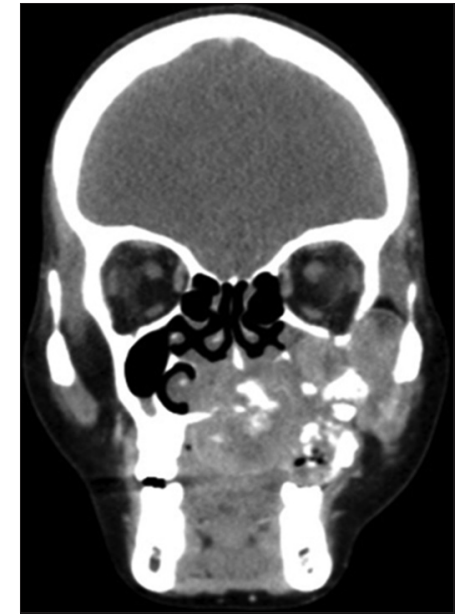

Fig. 3: Coronal contrast-enhanced computed tomography scan showing heterogeneously enhancing mass with destruction of bone in the hard palate and left maxilla which showed the presence of recurrence of the tumor. The tumor was excised with bilateral external carotid artery control. The patient reviewed 3 months with another recurrence but refused treatment and was lost to follow-up.

This "aggressive" variant of clear cell chondrosarcoma with frequent recurrence in the head and neck which is known for its good prognosis does not fit into literature and gives a take home message that we have to be prepared for eventual nonexistent prognosis in any tumor in the head and neck.

Consent: Patient consent has been obtained for reproduction of clinical images and data.

\section{REFERENCES}

1. Mokhtari S, Mirafsharieh A. Clear cell chondrosarcoma of the head and neck. Head Neck Oncol 2012;4:13.

2. Collins MS, Koyama T, Swee RG, Inwards CY. Clear cell chondrosarcoma: Radiographic, computed tomographic, and magnetic resonance findings in 34 patients with pathologic correlation. Skeletal Radiol 2003;32(12):687-94. 